\title{
Striking back
}

Stroke has disabled

millions of people,

stealing their ability to

walk or communicate.

Can future victims be

helped by treatments

that stimulate the

growth of new brain

cells? Alison Abbott

investigates.

$\mathrm{t}$ is cruel, capricious and a killer. In the United States alone, about 700,000 people

suffer from stroke every year, and up to $20 \%$ of them die within three months. Half are left disabled - many robbed of their power of speech, or their ability to walk or to manipulate objects.

Nevertheless, almost all stroke survivors regain at least some of their lost function over time. This is mostly achieved by their brains recruiting other neural circuits to take over the function of those that were damaged during the stroke, when a blood clot or a ruptured vessel starved part of the brain of oxygen. But in recent years, evidence has emerged that injured brains can also grow new neurons - contrary to decades-old biological dogma. And that has set neurologists thinking: suppose this natural mechanism could be supercharged, so that a stroke-damaged brain repaired itself.

The idea arose after neural stem cells were found in the adult brain. These can divide and differentiate into the two main classes of brain cell: neurons, which relay neural signals, and astrocytes, which provide a supportive environment for neurons to do their job.

\section{Self-help system}

Normally, the turnover of these stem cells is vanishingly low, and most of the differentiated cells produced are astrocytes. But in 2001, evidence emerged that traumatic brain injuries, such as those caused by a stroke, can stimulate stem cells to generate new neurons, at least in rodents ${ }^{2}$. These neurons have since been shown to migrate to the site of damage ${ }^{3-5}$. Left to itself, this mechanism is not up to the task of repairing gross damage caused by a stroke. But given the right tweaks and prompts, could it be made more efficient?

Stem cells capable of differentiating into neurons seem to exist mainly at two sites in

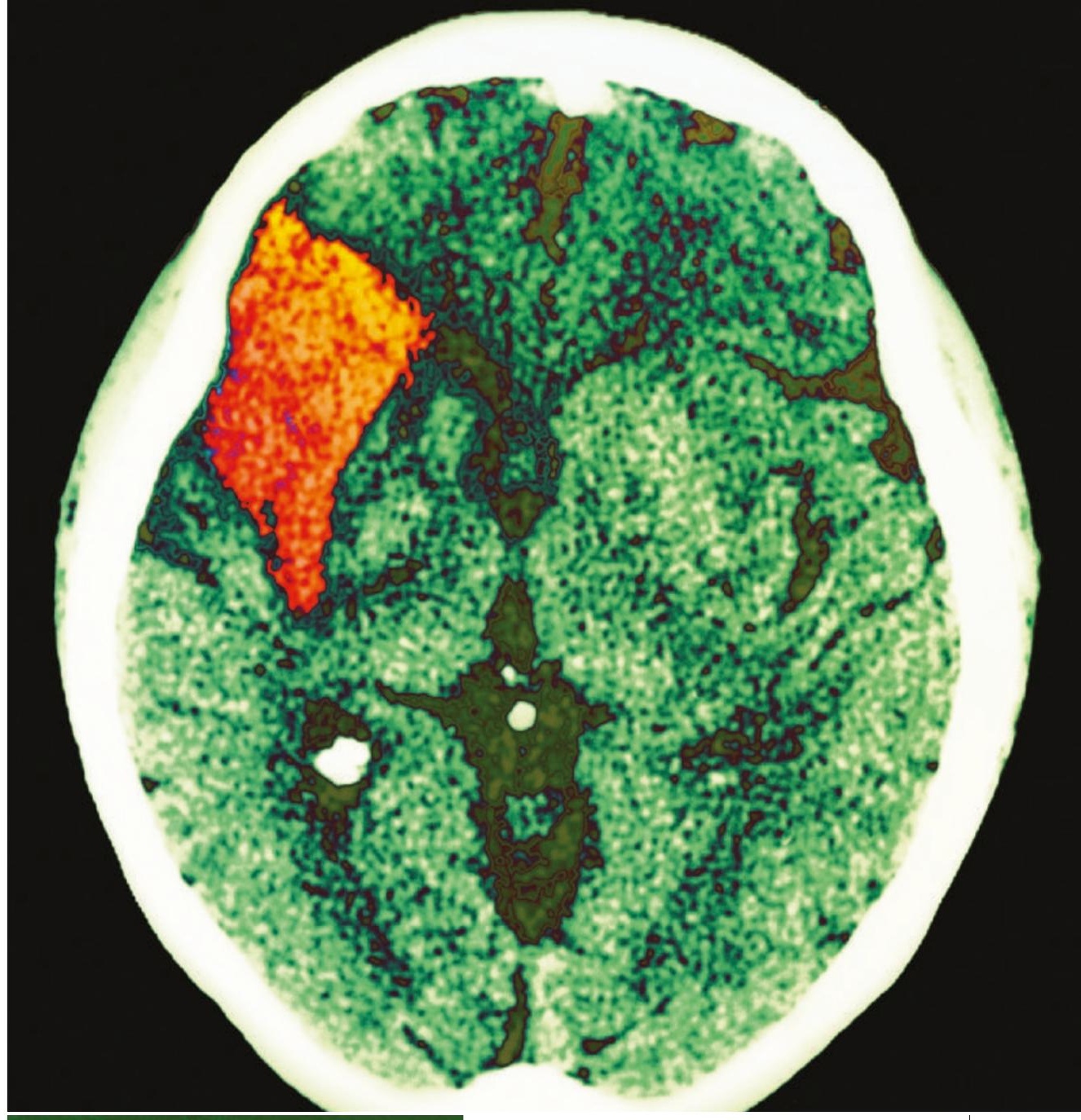

Repair mechanism: this brain scan shows the the extent of cell death (above, orange) caused by a stroke. Researchers have now discovered that, contrary to traditional belief, neural stem cells in the brain can generate replacement neurons (left, green) that will migrate to some injured sites.

Jack Parent of the University of Michigan Medical Center in Ann Arbor independently studied a rat model of stroke in which a brain area called the striatum, which lies close to the SVZ, was damaged by briefly blocking blood flow in the middle cerebral artery. The resulting damage stimulated the growth of new neurons in the SVZ, which travelled up to the damaged areas ${ }^{3,4}$.

\section{Limited response}

But the replacement of damaged cells was not extensive. Lindvall, for example, found that after a few weeks only $0.2 \%$ of the cells that had been destroyed were replaced, and that as many as $80 \%$ of the new neurons that had been produced had died $^{3}$. "But it showed that the self-repair response is there," he says.

At about the same time, Masato Nakafuku, then at the University of Tokyo, reported on his results using a different rat model of stroke. Nakafuku's team damaged the hippocampus by briefly interrupting the entire blood flow to the brain, and found that new neurons were produced in a nearby area, which migrated into the damaged region ${ }^{5}$. 


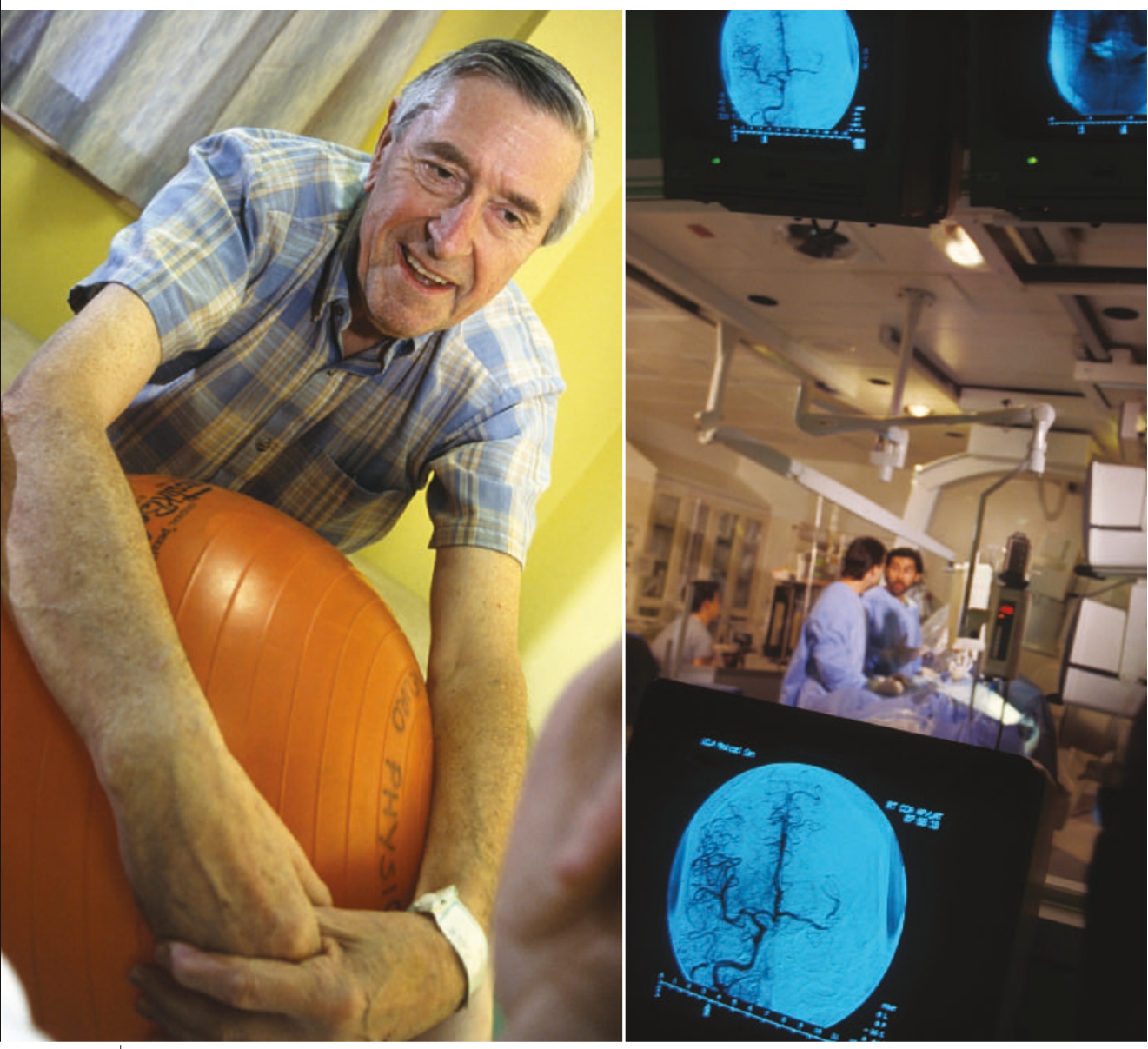

ث્山ે Physiotherapy (left) can aid recovery after treatment for a stroke (right), but do new neurons help too?

Most encouragingly, the group also showed that infusions into the brain of two growth factors, called EGF and FGF2, which cause stem cells to proliferate, greatly increased the number of new neurons produced - by almost ten fold in the case of EGF.

These new neurons formed connections with existing neuronal circuits. And the animals treated with growth factor seemed to recover some of the learning skills that they had lost immediately after their strokes. Nakafuku, who is now at the Cincinnati Children's Hospital Medical Center in Ohio, can't say for sure that this recovery was a result of the connections made by the new

"Antidepressants, cholesterol-lowering drugs and even Viagra have all been shown to induce the growth of new neurons." which has shown that irradiating rats to destroy stem cells before inducing stroke reduces the recovery of learning ${ }^{6}$, suggests that it may well be.

Encouraged by these results, many groups are now trying to enhance these effects. "Both the timing and duration of growthfactor infusion after stroke is critical," Nakafuku suggests. Parent adds: "We want to see if new, stroke-induced neurons integrate and function properly." That is an important issue, as the neurons that grow following epileptic seizures can integrate improperly into the brain's neural circuits, and actually help to ensure that symptoms continue ${ }^{7}$.

Researchers want to know why so few of the neurons generated after a stroke survive long-term - which will require a better understanding about the environment of the stroke-damaged brain. The inflammation associated with stroke could be to blame, says Lindvall. Certainly, inflammation impairs the normal turnover of new neurons in the hippocampus ${ }^{8,9}$.

It will also be important to induce the production of neurons in regions of the brain other than the striatum and hippocampus. So far, researchers have had little success in replacing neurons in the brain's cortex, which is frequently damaged by stroke. "But this may just be because we don't yet know how to do it," says Lindvall. Several research groups are now trying to develop animal models that will allow the cortex to be studied more precisely, he says.

David Greenberg of the Buck Institute in Novato, California, believes that important biological clues could come from studying animal models of neurodegenerative diseases such as Alzheimer's and Huntington's, in which it is now emerging that some of the dying neurons are replaced ${ }^{10,11}$. "Stroke is an acute insult and all sorts of things spill out as cells die en masse," Greenberg says. By studying the more sedate process of neuron damage and replacement that occurs in neurodegenerative diseases, he argues, it should be easier to understand the biological factors involved.

\section{Human touch}

Although much of the research into brain repair will continue to be done in rodent models, scientists would also like to observe the production of neurons in people. Several labs are trying to develop cell markers that could allow the differentiation of stem cells and the migration of new neurons to be tracked using positron emission tomography brain imaging. Such markers are likely to be structurally related to the building blocks of DNA, and so will be incorporated as dividing cells copy their genetic material.

In parallel to the work on basic biology, therapeutic leads are accumulating. Many different growth factors, in addition to EGF and FGF2, are being tested for their ability to promote the production of nerve cells. And because growth factors are proteins, which are difficult to administer as drugs and would have to be infused directly into the brain, researchers are also searching for smaller molecules with similar effects.

"There are plenty of candidates," says Alex Mercer, head of target discovery at the company NeuroNova in Stockholm, Sweden. Some therapeutics currently in use may provide a starting point for drug development. It is unclear whether these drugs will be as potent as growth factors, but antidepressants ${ }^{12}$, the cholesterol-lowering statins ${ }^{13}$ and even Viagra ${ }^{14}$ have all in the past couple of years been shown to induce the growth of new neurons.

Given the vast amount of work to be done on basic biology and drug development, researchers warn that it will be many years before hopes of a revolution in stroke therapy might be realized. "It is not that we are pessimistic," says Lindvall. "It's just that we need an awful lot of time to do the basic research that is necessary before we can think of approaching patients."

Alison Abbott is Nature's senior European correspondent.

\section{Gage, F. H. Science 287, 1433-1438 (2000).}

2. Jin, K. et al. Proc. Natl Acad. Sci. USA 98, 4710-4715 (2001).

3. Arvidsson, A., Collin, T., Kirik, D., Kokaia, Z. \& Lindvall, O. Nature Med. 8, 963-970 (2002).

4. Parent, J. M., Vexler, Z. S., Gong, C., Derugin, N. \& Ferriero, D. M. Ann. Neurol. 52, 802-813 (2002).

5. Nakatomi, H. et al. Cell 110, 429-441 (2002).

6. Raber, J. et al. Ann. Neurol. 55, 381-389 (2004).

7. Parent, J. M. Neuroscientist 9, 261-272 (2003).

8. Ekdahl, C. T., Claasen, J.-H., Bonde, S., Kokaia, Z. \& Lindvall, O. Proc. Natl Acad. Sci. USA 100, 13632-13637 (2003).

9. Monje, M. L., Toda, H. \& Palmer, T. D. Science 302, 1760-1765 (2003).

10. Curtis, M. A. et al. Proc. Natl Acad. Sci. USA 100, 9023-9027 (2003).

11. Jin, K. et al. Proc. Natl Acad. Sci. USA 101, 343-347 (2004).

12. Santarelli, L. et al. Science 301, 805-809 (2003).

13. Chen, J. et al. Ann. Neurol. 53, 743-751 (2003).

14. Zhang, R. et al. Stroke 33, 2675-2680 (2002). 